\title{
RFID Reader Localization Using Hard Decisions with Error Concealment
}

DOI:

10.1109/JSEN.2019.2914914

10.1109/JSEN.2019.2914914

\section{Document Version}

Accepted author manuscript

Link to publication record in Manchester Research Explorer

\section{Citation for published version (APA):}

Al-Jarrah, M., Al-Dweik, A., Alsusa, E., \& Damiani, E. (2019). RFID Reader Localization Using Hard Decisions with Error Concealment. IEEE Sensors Journal, 19(17), 7534 - 7542. https://doi.org/10.1109/JSEN.2019.2914914, https://doi.org/10.1109/JSEN.2019.2914914

\section{Published in:}

IEEE Sensors Journal

\section{Citing this paper}

Please note that where the full-text provided on Manchester Research Explorer is the Author Accepted Manuscript or Proof version this may differ from the final Published version. If citing, it is advised that you check and use the publisher's definitive version.

\section{General rights}

Copyright and moral rights for the publications made accessible in the Research Explorer are retained by the authors and/or other copyright owners and it is a condition of accessing publications that users recognise and abide by the legal requirements associated with these rights.

\section{Takedown policy}

If you believe that this document breaches copyright please refer to the University of Manchester's Takedown Procedures [http://man.ac.uk/04Y6Bo] or contact uml.scholarlycommunications@manchester.ac.uk providing relevant details, so we can investigate your claim.

\section{OPEN ACCESS}




\begin{abstract}
This paper presents an efficient reader localization algorithm in radio frequency identification (RFID) networks. In the proposed algorithm, $\left.{ }^{\left[R_{2}, 3\right.}\right]$ it is assumed that no channel state information is available at the reader and the backscatters of all RFID tags are converted to hard decisions using an energy detector. The accuracy of the estimated location is improved using an error concealment algorithm that utilizes the fact that adjacent tags are expected to produce similar decisions. The final location estimates are obtained using Particle Swarm Optimization of a maximum likelihood estimator that takes into account imperfections in the sensing and transmission processes. The performance of the proposed algorithm is evaluated in terms of the root mean square error using Monte Carlo simulation, and compared to other well-established localization algorithms. Moreover, Cramer-Rao lower bound is derived to assess the efficiency of the new estimator. The obtained results show that the proposed algorithm estimation accuracy is up to $26.5 \%$ more than the other benchmark estimators.
\end{abstract}

Index Terms-RFID, localization, positioning, location estimation, received signal strength.

\section{INTRODUCTION}

Radio frequency identification (RFID) technology plays a key role in a wide-range of industrial, commercial, medical, transportation and environmental applications. Indoor localization using RFID networks is a critical emerging application which has received extensive attention in the literature [1][12]. Generally speaking, the localization techniques reported in the literature aim at localizing the reader or one of the tags. In both cases, the required RFID infrastructure should include a reader and a grid of tags pre-deployed in the region of interest (RoI) [13]-[17]. For example, DiGiampaolo and Martinelli [5] proposed a system to localize a mobile robot equipped with RFID reader and odometry sensors with the tags fixed to the ceiling. The localization is based on measuring the phase of the signals transmitted by the tags with the aid of a multi-hypothesis Kalman filter. In [6], the influence of the tag interaction on the localization algorithm

M. Al-Jarrah, is with the Department of Electrical and Computer Engineering, Khalifa University of Science and Technology, Abu Dhabi, UAE. (E-mail: mohammad.aljarrah@ku.ac.ae).

A. Al-Dweik and E. Damiani are with Center on CyberPhysical Systems, Khalifa University, UAE. (E-mail: \{arafat.dweik, ernesto.damiani\}@ku.ac.ae)

E. Alsusa is with the School of Electrical and Electronic Engineering, University of Manchester, Manchester M13 9PL, U.K. (E-mail e.alsusa@manchester.ac.uk). is studied. A two-dimensional localization system for passive ultra-high frequency (UHF) RFID tags based evaluating the backscattered transponder signals is proposed in [7], where the phase and amplitude of signals are jointly used to provide accurate localization. The authors of [8] developed methods for the purpose of pattern matching to mitigate the effect of measurements' errors by clustering the tags and considering only a few at a time, denoted as the neighbors tags, in the localization process. In [9], localization and tracking of an RFID reader is proposed to achieve accurate localization using received signal strength indicator (RSSI) measurements obtained from multiple distributed passive tags. A new deployment optimization approach for readers with directional antennas is proposed in [12], where a novel particle swarm optimization (PSO) is applied. ${ }^{\left[R_{2,4}\right]} \mathrm{A}$ probabilistic model based on the recursive Kalman filter is considered in [10] to reduce errors in the least squares sense, while a Bayesian filter is applied in [14] using a fixed RF transmission power model to localize RFID tags. Moreover, hybrid RSSI and time of arrival (TOA) localization is considered in [16] to localize multiple targets, where an approximate solution for the positions is derived based on the weighted least squares criterion.

Although the aforementioned techniques offer high accuracy, their computational complexity and overhead are generally high. For example, the algorithms reported in [4][8], [12] employ TOA, time difference of arrival (TDOA), angle of arrival (AOA), or phase of arrival (POA). Such techniques require perfect time synchronization between all the transmitters and receivers, and accurate calculation for the entire cycle phase. Therefore, localization using RSSI can be considered as an efficient solution to reduce complexity, but it usually comes at the expense of reduced accuracy due to the impact of channel effects [6], [9], [18]. ${ }^{\left[R_{2,4}\right]} \mathrm{A}$ faulttolerant RFID reader localization approach, that can handle regional permanent faults is given in [2]. Although the proposed algorithm may provide reliable location estimates in certain scenarios, it is limited to dense distributions of passive tags, and its complexity is $O\left(n^{3}\right)$, where $n$ is the number of tags. To overcome the fading effects, localization based on hard decision (HD) RSSI is considered in [19] where a local voting algorithm (LVA) is used to correct the hard decisions of certain tags before estimating the reader's location. However, the estimator is designed under the assumption of error-free 
link between the tags and the reader, and the distances between the reader and neighbor tags are equal. Therefore, the LVA accuracy deteriorates significantly at low signal-to-noise ratios (SNRs). A simplified version of [19] is reported in [20], but without local voting, and hence, its performance is worse than LVA at high SNRs. ${ }^{\left[R_{2,2}\right]}$ In addition, a soft range limited $K$-nearest neighbors (KNNs) localization fingerprinting algorithm is proposed in [21], where a scale factor related to the physical distance between the user's previous position and reference location is considered.

$\left[R_{2,3}\right]$ To the best of the authors' knowledge, there is no work in the literature that tackled the RFID reader localization problem while considering imperfect transmission and error concealment. Therefore, we propose an efficient reader localization algorithm for RFID networks where it is assumed that some of the tags do not receive the reader's signal, and hence do not respond to the reader interrogation signal (RIS). Moreover, the algorithm is designed while considering the impact of channel fading and the fact that adjacent tags are not necessarily at equal distance from the reader. The localization process is based on the maximum likelihood (ML) principle combined with a low complexity error concealment process to provide accurate location estimates. The system performance is evaluated in terms of the root mean square error (RMSE) for which the Cramer-Rao lower bound (CRLB) is derived.

The rest of the paper is organized as follows. In Section II, the system model is presented, followed by the proposed localization algorithm in Section III. In Section IV, the CRLB of the proposed localization algorithm is derived. Section $\mathrm{V}$ presents the analytical and simulation results. Finally, conclusions are provided in Section VI.

\section{System Model and Problem Formulation}

Consider an RFID network that consists of one reader and $K$ tags distributed in the RoI with known positions. The reader sends its radio frequency (RF) interrogation signals at a particular frequency, and then listens to the return signals transmitted by the tags. A tag that detects the RIS responds by transmitting a confirmation signal back to the reader, otherwise it remains silent. As such, the reader and tags have two operating modes, the transmission and listening modes. To increase the probability of detecting its interrogation signals by the tags, the reader may send a sequence of $L$ signals to enable the tag to combine these signals before it decides to respond back to the reader. In the listening mode, the reader receives only $U \leq K$ signals, where $U$ is the number of tags that have successfully detected the RIS. The $K-U$ tags do not respond because they are outside the coverage range of the reader, or due to channel fading and/or system noise. The reader's objective is to determine its location based on the received $U$ signals.

\section{A. Received signal model at the tag}

The baseband representation of the received signal at the $k$ th tag during the $l$ th signaling period can be written as

$S_{k}[l]=\sqrt{P_{R} \alpha_{k}} \hbar_{k}[l]+n_{k}[l], k=[1,2, \ldots, K], l=[1,2, \ldots, L]$

where $P_{R}$ is the reader transmit power, $\hbar_{k} \sim \mathcal{C N}\left(0, \sigma_{\hbar}^{2}\right)$ is a complex Gaussian distributed channel gain, $n_{k} \sim$ $\mathcal{N}\left(0, N_{0} / 2\right)$ is the additive white Gaussian noise (AWGN) and $\alpha_{k}$ is the free space path loss,

$$
\alpha_{k}=\left(\frac{\lambda}{4 \pi d_{k}}\right)^{2} \text {. }
$$

The wavelength of the RIS in (2) is denoted as $\lambda$ and the distance between the $k$ th tag and the reader is denoted as $d_{k}$, which can be computed as

$$
d_{k}=\sqrt{\left(x_{G_{k}}-x_{R}\right)^{2}+\left(y_{G_{k}}-y_{R}\right)^{2}}
$$

where $\left(x_{R}, y_{R}\right)$ and $\left(x_{G_{k}}, y_{G_{k}}\right)$ are the Cartesian coordinates of the reader and $k$ th tag, respectively.

To mitigate the impact of channel fading and noise, the participant tags may take repeated votes before deciding to respond to the RIS. Under power and delay constraints, the polling frequency $L$ may be adaptable to minimize the probability of error [22]. However, such optimization could be computationally prohibitive in RFID scenarios due to computational power constraints, and thus, this work considers a fixed $L$ for all tags. Given that the reader sends a sequence of $L$ polling signals during its transmission mode that last for $T_{R}$ seconds, combining the $L$ RISs can be performed using various techniques, however equal gain combining is adopted in this context due to its simple implementation. Therefore, the combined $L$ RISs can be written as

$$
\begin{aligned}
S_{k} & =\frac{1}{L} \sum_{l=1}^{L} \sqrt{P_{R} \alpha_{k}} \rho_{k}[l]+n_{k}[l] \\
& =A_{k}+w_{k}
\end{aligned}
$$

where $A_{k}=\frac{1}{L} \sqrt{P_{R} \alpha_{k}} \sum_{l=1}^{L} \rho_{k}[l], w_{k} \sim \mathcal{N}\left(0, \sigma_{w}^{2}\right)$, and $\sigma_{w}^{2}=N_{0} / 2 L$. For large values of $L$, the ensemble average can be approximated by the statistical average, and thus $A_{k} \approx \sqrt{P_{R} \alpha_{k}} \mathcal{E}\left\{\rho_{k}[l]\right\}$, where $\mathcal{E}\{\cdot\}$ represents the expectation process. Finally, the signal $S_{k}$ is applied to the detector, which makes a binary decision (hard decision) whether to respond to the RIS or not. The decision at the tag can be described by

$$
u_{k}=\left\{\begin{array}{ll}
0, & S_{k}<\tau_{k} \\
1, & S_{k} \geq \tau_{k}
\end{array} .\right.
$$

The detection threshold $\tau_{k}$ that can be selected to control the tags' sensitivity to the received signals. More specifically, $\tau_{k}$ can be adjusted to limit the number of tags that may receive and respond to the RIS. By noting that $A_{k} \approx \sqrt{P_{R} \alpha_{k}} \mathcal{E}\left\{\rho_{k}[l]\right\}$ 
where $d_{0}$ is the radius of the reader coverage area. ${ }^{\left[R_{1,1 a}\right]}$ It is worth noting that the effect of the free space path loss in (6) is based on the assumption that the radiation pattern of the reader is circular. Although it might be infeasible to design perfectly circular radiation patterns in practice, there are several antennas designed for RFID applications that has near-ideal omnidirectional antennas [25]-[27]. Consequently, the reader radiation pattern can be closely approximated by a circular pattern, and the free space path loss for all tags at a distance $d_{0}$ from the reader can be considered equivalent.

Based on (4) and (5), the probability that a tag successfully detects the RIS is given by

$$
\operatorname{Pr}\left(u_{k}=1\right)=Q\left(\frac{\tau_{k}-A_{k}}{\sigma_{w}}\right)
$$

where $Q(\cdot)$ is the Q-function. After the detection process, each tag with $u_{k}=1$ will respond back to the reader by sending a continuous signal with average transmit power $P_{G}$ and duration of $T_{G}$ seconds.

\section{B. Received signal model at the reader}

${ }^{\left[R_{1,1 b}\right]}$ In this work, it is assumed that the RFID system employs an anti-collision protocol, and hence there is no interference between the signals transmitted by the $U$ active tags [4], [6]. Therefore, the received signal from the $k$ th tag can be written as

$$
y_{k}=\sqrt{P_{G}} \hbar_{k}[l] \sqrt{\alpha_{k}} u_{k}+\varphi_{k}
$$

where $\hbar_{k}[l] \sqrt{\alpha_{k}} \triangleq h_{k} \sim \mathcal{C N}\left(0, \sigma_{\hbar}^{2} \alpha_{k}\right)$ is the overall channel coefficient that captures the small and large scale fading effects, and $\varphi_{k} \sim \mathcal{C N}\left(0, \sigma_{\varphi}^{2}\right)$ is the AWGN. Thus, $y_{k}$ can be written as

$$
y_{k}=\sqrt{P_{G}} h_{k} u_{k}+\varphi_{k} .
$$

To estimate its location, defined by $\boldsymbol{\theta} \triangleq\left[x_{R}, y_{R}\right]$, the reader initially detects the responses collected from the $K$ tags. To avoid the channel estimation overheads for $K$ signals, the reader may use blind detection schemes such as energy detection [18], where the received signal energy can be expressed as

$$
r_{k}=\left|y_{k}\right|^{2}=\left|\sqrt{P_{G}} h_{k} u_{k}+\varphi_{k}\right|^{2} .
$$

Based on (10), the ML estimator (MLE) using $\mathbf{r}=$ $\left[r_{1}, r_{2}, \ldots, r_{K}\right]$ is given by

$$
\begin{aligned}
\hat{\boldsymbol{\theta}} & =\arg \max _{\boldsymbol{\theta}} \sum_{k=1}^{K} \ln f\left(r_{k} \mid \boldsymbol{\theta}\right) \\
& =\arg \max _{\boldsymbol{\theta}} \sum_{k=1}^{K} \ln \left(\sum_{u_{k}=0}^{1} f\left(r_{k} \mid u_{k}\right) \operatorname{Pr}\left(u_{k} \mid \boldsymbol{\theta}\right)\right)(11)
\end{aligned}
$$

where

$$
\operatorname{Pr}\left(u_{k} \mid \boldsymbol{\theta}\right)=1-u_{k}+(-1)^{u_{k}+1} Q\left(\frac{\tau_{k}-A_{k}}{\sigma_{w}}\right) .
$$

It is worth noting that $\boldsymbol{\theta}$ in (12) is implicitly included in $A_{k}$. Since $y_{k} \sim \mathcal{C N}\left(0, P_{G} u_{k}^{2} \sigma_{h}^{2}+\sigma_{\varphi}^{2}\right)$, then $r_{k}$ is exponentially distributed with mean $\beta_{u_{k}}=P_{G} u_{k}^{2} \sigma_{h}^{2}+\sigma_{\varphi}^{2}$, where $\sigma_{h}^{2}=$ $\sigma_{\hbar}^{2} \alpha_{k}$. Consequently, the MLE can be written as

$\hat{\boldsymbol{\theta}}_{\mathrm{SDE}}=\arg \max _{\boldsymbol{\theta}} \sum_{k=1}^{K} \ln \left(\sum_{u_{k}=0}^{1} \frac{1}{\beta_{u_{k}}} \exp \left(\frac{-r_{k}}{\beta_{u_{k}}}\right) \operatorname{Pr}\left(u_{k} \mid \boldsymbol{\theta}\right)\right)$.

Because the MLE in (13) is based on the unquantized values, i.e. soft values, of $r_{k}$, it is denoted as soft decision estimator (SDE) [18]. As can be noted from (13), it is infeasible to compute $\hat{\boldsymbol{\theta}}$ analytically, and thus, exhaustive search methods should be used.

An alternative approach to estimate the location of the reader is to use $r_{k}$ to generate hard decisions (binary decisions), for each tag individually, and then the likelihood function is derived based on the hard decisions, and hence it is denoted as the HD estimator (HDE) [20]. In other words, the reader tries to estimate $u_{k} \forall k$, and use the estimated values, denoted as $\hat{\mathbf{u}}=\left[\hat{u}_{1}, \hat{u}_{2}, \ldots, \hat{u}_{K}\right]$ to estimate its location. The optimum HD detector can be formulated as,

$$
\begin{aligned}
\hat{u}_{k} & =\arg \max _{u_{k} \in\{0,1\}} f\left(r_{k} \mid u_{k}\right), k=\{1,2, \ldots, K\} \\
& =\arg \min _{u_{k} \in\{0,1\}} \frac{r_{k}}{\beta_{u_{k}}}+\ln \beta_{u_{k}} .
\end{aligned}
$$

After some manipulations, (14) can be written as

$$
\hat{u}_{k}=\left\{\begin{array}{cc}
0, & \gamma_{0} \leq r_{k} \leq \gamma_{1} \\
1, & \gamma_{1} \leq r_{k} \leq \gamma_{2}
\end{array}\right.
$$

where $\gamma_{0}=0, \gamma_{2}=\infty$ and

$$
\gamma_{1}=\frac{\ln \beta_{1}-\ln \beta_{0}}{\beta_{1}-\beta_{0}} \beta_{1} \beta_{0} .
$$

Thereafter, the MLE based on $\hat{\mathbf{u}}$ can be formulated as

$$
\hat{\boldsymbol{\theta}}_{\mathrm{HDE}}=\arg \max _{\boldsymbol{\theta}} \sum_{k=1}^{K} \ln \left(\sum_{u_{k} \in\{0,1\}} \operatorname{Pr}\left(\hat{u}_{k} \mid u_{k}\right) \operatorname{Pr}\left(u_{k} \mid \boldsymbol{\theta}\right)\right) .
$$

The pairwise probability $\operatorname{Pr}\left(\hat{u}_{k} \mid u_{k}\right)$ can be derived from (15) and (16),

$$
\operatorname{Pr}\left(\hat{u}_{k}=n \mid m\right)=e^{-\frac{\gamma_{n}}{\beta_{m}}}-e^{-\frac{\gamma_{n+1}}{\beta_{m}}}
$$

where $\{n, m\} \in\{0,1\}$.

In high signal to noise ratio (SNR) scenarios, the channel can be considered error free and $\operatorname{Pr}\left(u_{k} \mid \boldsymbol{\theta}\right)$ is equal for nearby tags. Consequently, the decisions made by a particular tag can be corrected at the reader based on the decisions of the nearest $M$ tags [19], and hence, this approach is denoted as the local voting algorithm (LVA). Given that the set of all tags in the RoI is denoted as $\mathbb{A}$, and the set of $M$ neighboring tags is denoted 
as $\mathbb{B}$, where $\mathbb{B} \subseteq \mathbb{A}$, the decision correction process for each signal in $\mathbb{B}$ can be described as

$$
\tilde{u}_{k}= \begin{cases}1, & \mathcal{W}\left(\mathbf{u}_{\mathbb{B}}\right) \geq \epsilon_{0} \\ 0, & \mathcal{W}\left(\mathbf{u}_{\mathbb{B}}\right)<\epsilon_{0}\end{cases}
$$

where $\mathbf{u}_{\mathbb{B}}=\left[u_{1}, u_{2}, \ldots, u_{M}\right], \mathcal{W}(\cdot)$ is the Hamming weight, $\tilde{u}_{k}$ is the corrected decision and $\epsilon_{0} \triangleq\lceil M / 2\rceil$, where $\lceil\cdot\rceil$ is the ceiling function. Therefore, the corrected decisions have the following probabilities,

$$
\begin{aligned}
\operatorname{Pr}\left(\tilde{u}_{k}=1 \mid \boldsymbol{\theta}\right)=\sum_{i=\left\lceil\epsilon_{0}\right\rceil}^{M}\left(\begin{array}{c}
M \\
i
\end{array}\right)\left(\operatorname{Pr}\left(u_{k}=1 \mid \boldsymbol{\theta}\right)\right)^{i} \\
\times\left(1-\operatorname{Pr}\left(u_{k}=1 \mid \boldsymbol{\theta}\right)\right)^{M-i}
\end{aligned}
$$

and $\operatorname{Pr}\left(\tilde{u}_{k}=0 \mid \boldsymbol{\theta}\right)=1-\operatorname{Pr}\left(\tilde{u}_{k}=1 \mid \boldsymbol{\theta}\right)$. Therefore, the MLE based on the LVA algorithm is

$$
\hat{\boldsymbol{\theta}}_{\mathrm{LVA}}=\arg \max _{\boldsymbol{\theta}} \sum_{k=1}^{K} \ln \left(\operatorname{Pr}\left(\tilde{u}_{k} \mid \boldsymbol{\theta}\right)\right) .
$$

${ }^{\left[R_{1,2}\right]}$ Generally speaking, the signal transmitted/received by the tag depends on the tag model and frequency band used. In this work, we consider that all RFID tags are omnidirectional in both horizontal and vertical dimensions. Therefore, the transmitted/received signal models of the tags are independent of the tag orientation. The design of such tags at $925 \mathrm{MHz}$ frequency is reported in [28].

\section{The Proposed Localization Algorithm}

To maintain low complexity of the MLE with hard decisions, the proposed algorithm is based on binary energy detection as the first stage to produce the vector $\hat{\mathbf{u}}$ as described in (15). In the second stage, error concealment is applied to correct the erroneous decisions and produce a new set of decisions denoted as $\tilde{\mathbf{u}}$. Finally, the MLE is applied to estimate the location of the reader. The proposed localization algorithm with error concealment (LEC) and the MLE are derived in the following two subsections.

\section{A. The error concealment process}

Due to channel impairments, it is highly likely that $\hat{\mathbf{u}}_{\mathbb{B}} \neq$ $\mathbf{u}_{\mathbb{B}}$, and hence the performance of the MLE may deteriorate. In practical scenarios, tags in the vicinity of each other are expected to produce similar decisions, i.e., $u_{1}=u_{2}=\cdots=$ $u_{M}$. Therefore, the LEC is designed to exploit such correlation to correct the errors before the location estimation process. An example for the LEC using $M=9$ is given in Algorithm 1.

The correction threshold $\epsilon$ should be dynamically adjusted to consider the variable number of tags in $\mathbb{B}$ that detected the RIS as well as the imbalanced probability induced by the hard decision detector. By noting that $\epsilon$ is typically set to $\epsilon_{0}$ in error free transmissions [19], it can be dynamically changed by considering the channel effects on the signals sent from the

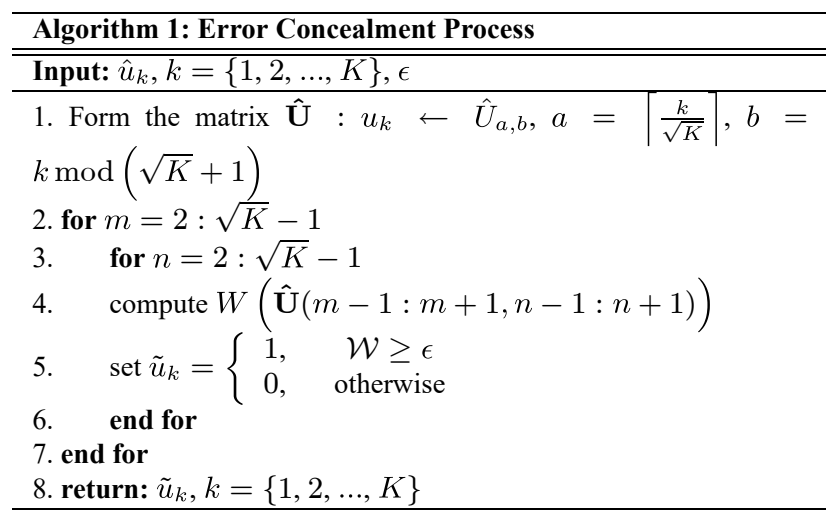

tags to the reader. Thus,

$$
\begin{aligned}
\epsilon & =\mathcal{E}\left[\mathcal{W}\left(\hat{\mathbf{u}}_{\mathbb{B}}\right) \mid \mathcal{W}\left(\mathbf{u}_{\mathbb{B}}\right)=\epsilon_{0}\right] \\
& =\sum_{i=0}^{M} \mathcal{E}\left[\hat{u}_{i} \mid \mathcal{W}\left(\mathbf{u}_{\mathbb{B}}\right)=\epsilon_{0}\right] \\
& =\sum_{i=0}^{M} \sum_{\hat{u}_{i}=\{0,1\}} \hat{u}_{i} \operatorname{Pr}\left(\hat{u}_{i} \mid \mathcal{W}\left(\mathbf{u}_{\mathbb{B}}\right)=\epsilon_{0}\right) .
\end{aligned}
$$

By noting that $\hat{u}_{i} \operatorname{Pr}\left(\hat{u}_{i} \mid \epsilon_{0}\right)=0$ when $\hat{u}_{i}=0$, then (22) can be simplified to

$$
\epsilon=\sum_{i=0}^{M} \operatorname{Pr}\left(\hat{u}_{i}=1 \mid \mathcal{W}\left(\mathbf{u}_{\mathbb{B}}\right)=\epsilon_{0}\right) .
$$

Using the law of total probability, the summand in (23) can be written as

$$
\begin{aligned}
& \operatorname{Pr}\left(\hat{u}_{k}=1 \mid \mathcal{W}\left(\mathbf{u}_{\mathbb{B}}\right)=\epsilon_{0}\right)= \\
& \quad \operatorname{Pr}\left(u_{k}=1 \mid \mathcal{W}\left(\mathbf{u}_{\mathbb{B}}\right)=\epsilon_{0}\right) \operatorname{Pr}\left(\hat{u}_{k}=1 \mid u_{k}=1\right) \\
& +\operatorname{Pr}\left(u_{k}=0 \mid \mathcal{W}\left(\mathbf{u}_{\mathbb{B}}\right)=\epsilon_{0}\right) \operatorname{Pr}\left(\hat{u}_{k}=1 \mid u_{k}=0\right) .
\end{aligned}
$$

Given that $\mathcal{W}\left(\mathbf{u}_{\mathbb{B}}\right)=\epsilon_{0}$, then $\operatorname{Pr}\left(u_{k}=1 \mid \mathcal{W}\left(\mathbf{u}_{\mathbb{B}}\right)=\epsilon_{0}\right)=$ $\frac{\epsilon_{0}}{M}$, and thus

$$
\begin{array}{r}
\operatorname{Pr}\left(\hat{u}_{k}=1 \mid \mathcal{W}\left(\mathbf{u}_{\mathbb{B}}\right)=\epsilon_{0}\right)=\frac{\epsilon_{0}}{M} \operatorname{Pr}\left(\hat{u}_{k}=1 \mid u_{k}=1\right) \\
+\left(1-\frac{\epsilon_{0}}{M}\right) \operatorname{Pr}\left(\hat{u}_{k}=1 \mid u_{k}=0\right)
\end{array}
$$

where $\operatorname{Pr}\left(\hat{u}_{k}=1 \mid u_{k}=1\right)$ and $\operatorname{Pr}\left(\hat{u}_{k}=1 \mid u_{k}=0\right)$ are given in (18).

In the special case that the average SNRs of the received signals from the tags in $\mathbb{B}$ are equal, then the probability of error for all tags in $\mathbb{B}$ is equal, which is mostly the case since all tags in $\mathbb{B}$ have approximately the same path loss. Moreover, by noting that for $\lceil M / 2\rceil \approx M / 2$ for $M \gg 1$, then $\epsilon_{0} / M \approx$ 0.5 . By substituting $\epsilon_{0} / M=0.5$ in (25), and substituting (25) in (23), then $\epsilon$ can be computed as

$$
\epsilon=\frac{M}{2}\left(e^{-\left(\frac{\gamma_{1}}{\beta_{1}}\right)}+e^{-\left(\frac{\gamma_{1}}{\beta_{0}}\right)}\right) .
$$

Fig. 1 shows an example selected from one of the simulation 


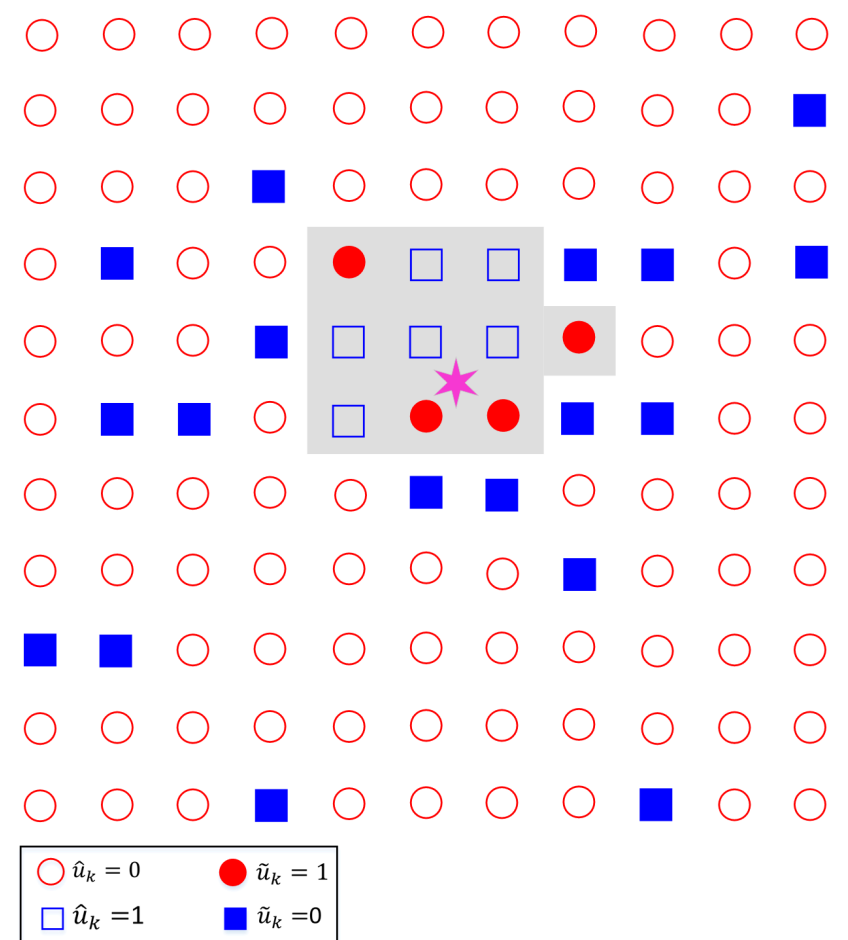

Fig. 1. Example of the error concealment process for $M=9$.

result for the error concealment process for an $11 \times 11$ grid. The sliding window started from the top-left corner where $M=9$ and $\epsilon=5$. As can be noted from the figure, the concealment process inverted 22 values, 18 tags were excluded $(1 \rightarrow 0)$ and 4 were included $(0 \rightarrow 1)$. In this scenario, about 4 of the excluded tags are close to the reader and should have been considered. nevertheless, the number of eliminated outliers is much larger than the number of legitimate tags that were erroneously excluded, which implies that the localization accuracy will eventually improve.

1) LEC based maximum likelihood estimator: The MLE is formulated based on the corrected decisions obtained from the LEC, and thus

$$
\hat{\boldsymbol{\theta}}_{\mathrm{LEC}}=\arg \max _{\boldsymbol{\theta}} \sum_{k=1}^{K} \ln \left(\operatorname{Pr}\left(\tilde{u}_{k} \mid \boldsymbol{\theta}\right)\right) .
$$

By noting that $\tilde{u}_{k} \in\{0,1\}$, the summand in (27) $\sum_{k=1}^{K} \ln \left(\operatorname{Pr}\left(\tilde{u}_{k} \mid \boldsymbol{\theta}\right)\right) \triangleq \Lambda_{\mathrm{LEC}}(\boldsymbol{\theta})$ can be expressed as

$$
\begin{aligned}
\Lambda_{\mathrm{LEC}}(\boldsymbol{\theta})=\sum_{k=1}^{K} \tilde{u}_{k} \ln & \left(\operatorname{Pr}\left(\tilde{u}_{k}=1 \mid \boldsymbol{\theta}\right)\right) \\
& +\left(1-\tilde{u}_{k}\right) \ln \left(\operatorname{Pr}\left(\tilde{u}_{k}=0 \mid \boldsymbol{\theta}\right)\right) .
\end{aligned}
$$

Since $\mathcal{W}\left(\hat{\mathbf{u}}_{\mathbb{B}}\right)$ is a sum of $M$ independent Bernoulli random variables with different probability of success, then $\tilde{u}_{k}$ is a Poisson-binomial distributed random variable with a cumula-

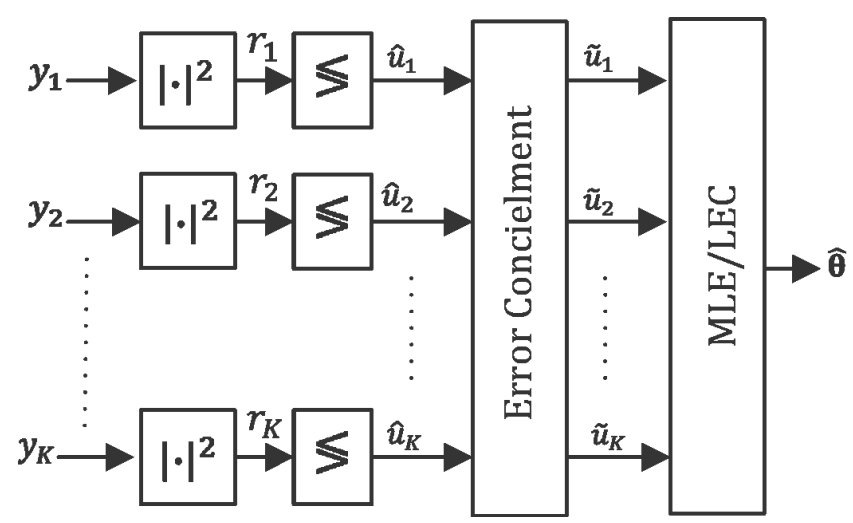

Fig. 2. Block Diagram of the proposed LEC.

\begin{tabular}{l}
\hline \multicolumn{1}{c}{ Algorithm 2: Proposed LEC } \\
\hline \hline Input: $y_{k}, k=\{1,2, \ldots, K\}$ \\
\hline 1. for $k=1: K$ \\
2. Compute $r_{k}$ using $((10)$ \\
3. Compute $\hat{u}_{k}$ using $(15)$ \\
4. end for \\
5. Compute $\tilde{\mathbf{u}}=\left[\tilde{u}_{1}, \tilde{u}_{2}, \ldots, u_{K}\right]$ using Algorithm 1 \\
6. for $k=1: K$ \\
7. Compute $p_{l}$ using (30) \\
8. Compute $\operatorname{Pr}\left(\tilde{u}_{k}=0 \mid \boldsymbol{\theta}\right)$ using (29) \\
9. end for \\
10. Compute $\Lambda_{\mathrm{LEC}}(\boldsymbol{\theta})$ using (28) \\
11. Compute $\hat{\boldsymbol{\theta}}$ in $(27)$ using PSO \\
12. return $\hat{\boldsymbol{\theta}}=\left[\hat{x}_{R}, \hat{y}_{R}\right]$ \\
\hline
\end{tabular}

tive distribution function (CDF) given by [23]

$$
\begin{aligned}
\operatorname{Pr}\left(\tilde{u}_{k}=0 \mid \boldsymbol{\theta}\right)=\operatorname{Pr}\left(\mathcal{W}\left(\hat{\mathbf{u}}_{\mathbb{B}}\right)<\epsilon \mid \boldsymbol{\theta}\right) & \\
=\frac{\epsilon}{M+1}+ & \frac{1}{M+1} \sum_{i=1}^{M}\left(\frac{1-e^{-\frac{j 2 \pi i \epsilon}{M+1}}}{1-e^{-\frac{j 2 \pi i}{M+1}}}\right. \\
& \left.\quad \times \prod_{l=1}^{M}\left(p_{l} e^{\frac{j 2 \pi i}{M+1}}+1-p_{l}\right)\right)
\end{aligned}
$$

where $p_{l}$ is given by

$$
\begin{aligned}
p_{l} & =\operatorname{Pr}\left(\hat{u}_{l}=1 \mid \boldsymbol{\theta}\right) \\
& =\sum_{u_{l} \in\{0,1\}} \operatorname{Pr}\left(\hat{u}_{l}=1 \mid u_{l}\right) \operatorname{Pr}\left(u_{l} \mid \boldsymbol{\theta}\right) .
\end{aligned}
$$

Finally, (29) is substituted in (28), and PSO is applied to compute $\hat{\boldsymbol{\theta}}=\left(\hat{x}_{R}, \hat{y}_{R}\right)$ which maximizes $\Lambda_{\mathrm{LEC}}(\boldsymbol{\theta})$.

To summarize the proposed system, Fig. 2 and Algorithm 2 are presented, where the figure shows the system level block diagram while Algorithm 2 describes the system in a step-bystep manner.

Generally speaking, all HD based estimators [18]-[20] have comparable computational complexity, which is mostly determined by the maximization of the likelihood function. However, the proposed LEC and LVA [19] have some ad- 
ditional complexity over [20] caused by the error correction process. Nevertheless, the correction process is based on a low complexity majority voting operation within a small size sliding window, and hence, the computational complexity of the proposed LEC and [18]-[20] can be considered equivalent.

\section{CRLB of the Proposed Estimator}

The variance of an unbiased estimator is bounded by $\mathbf{F}^{-1}$, where $\mathbf{F}$ is the Fisher information matrix [24],

$$
\mathcal{E}\left[(\hat{\boldsymbol{\theta}}-\boldsymbol{\theta})(\hat{\boldsymbol{\theta}}-\boldsymbol{\theta})^{H}\right] \geq \mathbf{F}^{-1}
$$

where $(\cdot)^{H}$ is the Hermitian operator. The Fisher information matrix of $\hat{\boldsymbol{\theta}}=\max _{\boldsymbol{\theta}} \Lambda_{\mathrm{LEC}}(\boldsymbol{\theta})$ is given by

$$
\begin{aligned}
\mathbf{F} & =\mathcal{E}\left[-\nabla_{\boldsymbol{\theta}}\left(\nabla_{\boldsymbol{\theta}}\right)^{t}\left(\Lambda_{\mathrm{LEC}}(\boldsymbol{\theta})\right)\right] \\
& =-\mathcal{E}\left[\begin{array}{cc}
\frac{\partial^{2} \Lambda_{\mathrm{LEC}}(\boldsymbol{\theta})}{\partial x_{R}^{2}} & \frac{\partial^{2} \Lambda_{\mathrm{LEC}}(\boldsymbol{\theta})}{\partial x_{R} \partial y_{R}} \\
\frac{\partial^{2} \Lambda_{\mathrm{LEC}}(\boldsymbol{\theta})}{\partial x_{R} \partial y_{R}} & \frac{\partial^{2} \Lambda_{\mathrm{LEC}}(\boldsymbol{\theta})}{\partial y_{R}^{2}}
\end{array}\right]
\end{aligned}
$$

where $\nabla_{\boldsymbol{\theta}}$ and $(\cdot)^{t}$ are the gradient and transpose operators, respectively. The elements of $\mathbf{F}$ are

$$
\begin{gathered}
\mathbf{F}_{1,1}=\sum_{k=1}^{K} \sum_{\tilde{u}_{k}} \frac{1}{\operatorname{Pr}\left(\tilde{u}_{k} \mid \boldsymbol{\theta}\right)}\left(\frac{\partial}{\partial x_{R}} \operatorname{Pr}\left(\tilde{u}_{k} \mid \boldsymbol{\theta}\right)\right)^{2} \\
\mathbf{F}_{2,2}=\sum_{k=1}^{K} \sum_{\tilde{u}_{k}} \frac{1}{\operatorname{Pr}\left(\tilde{u}_{k} \mid \boldsymbol{\theta}\right)}\left(\frac{\partial}{\partial y_{R}} \operatorname{Pr}\left(\tilde{u}_{k} \mid \boldsymbol{\theta}\right)\right)^{2} \\
\mathbf{F}_{1,2}=\sum_{k=1}^{K} \sum_{\tilde{u}_{k}} \frac{1}{\operatorname{Pr}\left(\tilde{u}_{k} \mid \boldsymbol{\theta}\right)} \frac{\partial}{\partial x_{R}} \operatorname{Pr}\left(\tilde{u}_{k} \mid \boldsymbol{\theta}\right) \frac{\partial}{\partial y_{R}} \operatorname{Pr}\left(\tilde{u}_{k} \mid \boldsymbol{\theta}\right) \\
\mathbf{F}_{2,1}=\mathbf{F}_{1,2}
\end{gathered}
$$

and the derivative $\frac{\partial}{\partial x_{R}} \operatorname{Pr}\left(\tilde{u}_{k} \mid \boldsymbol{\theta}\right)$ is given by

$$
\begin{gathered}
\frac{\partial}{\partial x_{R}} \operatorname{Pr}\left(\tilde{u}_{k}=1 \mid \boldsymbol{\theta}\right)=\frac{-1}{M+1} \sum_{i=1}^{M}\left(\frac{1-e^{-\frac{j 2 \pi i \epsilon}{M+1}}}{1-e^{-\frac{j 2 \pi i}{M+1}}}\right. \\
\left.\times \prod_{l=1}^{M}\left(p_{l} e^{\frac{j 2 \pi i}{M+1}}+1-p_{l}\right) \times \sum_{l=1}^{M} \frac{\partial p_{l}}{\partial x_{R}} \frac{\left(e^{\frac{j 2 \pi i}{M+1}}-1\right)}{p_{l} e^{\frac{j 2 \pi i}{M+1}}+1-p_{l}}\right)
\end{gathered}
$$

where $j \triangleq \sqrt{-1}$, and

$$
\begin{aligned}
\frac{\partial p_{l}}{\partial x_{R}} & =\frac{-\sqrt{P_{R}} \mathcal{E}\left\{\rho_{k}[l]\right\} \lambda d_{l}^{-3}}{4 \pi \sigma_{l} \sqrt{2 \pi}} e^{-\frac{\left(\tau_{l}-A_{l}\right)^{2}}{2 \sigma_{l}^{2}}}\left(x_{R}-x_{G_{k}}\right) \\
& \times\left[\operatorname{Pr}\left(\hat{u}_{l}=1 \mid u_{l}=1\right)-\operatorname{Pr}\left(\hat{u}_{l}=1 \mid u_{l}=0\right)\right] .
\end{aligned}
$$

It can be noted that $\frac{\partial}{\partial x_{R}} \operatorname{Pr}\left(\tilde{u}_{k}=0 \mid \boldsymbol{\theta}\right)=$ $-\frac{\partial}{\partial x_{R}} \operatorname{Pr}\left(\tilde{u}_{k}=1 \mid \boldsymbol{\theta}\right) \quad$ and $\quad \frac{\partial}{\partial y_{R}} \operatorname{Pr}\left(\tilde{u}_{k}=0 \mid \boldsymbol{\theta}\right) \quad=$ $-\frac{\partial}{\partial y_{R}} \operatorname{Pr}\left(\tilde{u}_{k}=1 \mid \boldsymbol{\theta}\right) \quad$ since $\operatorname{Pr}\left(\tilde{u}_{k}=0 \mid \boldsymbol{\theta}\right)=1-$ $\operatorname{Pr}\left(\tilde{u}_{k}=1 \mid \boldsymbol{\theta}\right)$. Moreover, $\left(x_{R}-x_{G_{k}}\right)$ in (37) and (38) can be replaced by $\left(y_{R}-y_{G_{k}}\right)$ to find $\frac{\partial}{\partial y_{R}} \operatorname{Pr}\left(\tilde{u}_{k} \mid \boldsymbol{\theta}\right)$.

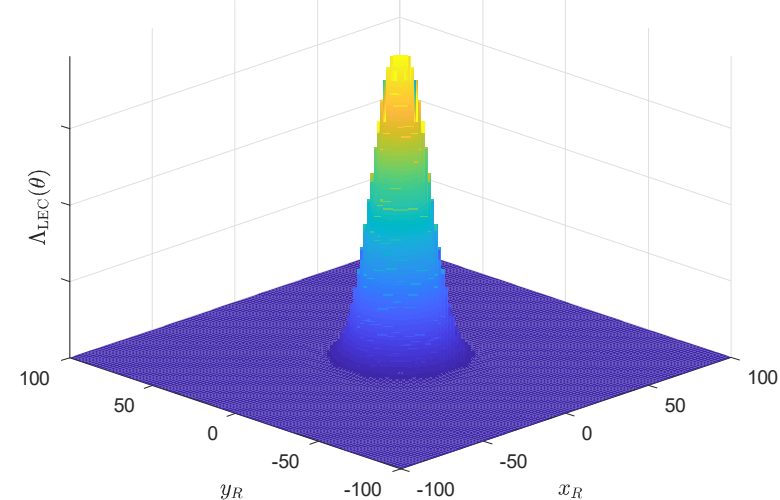

Fig. 3. The log-likelihood function where $K=256, L=50, \mathrm{SNR}=50$ $\mathrm{dB}$ and $\left(x_{R}, y_{R}\right)=(0,0)$.

\section{NUMERIC RESULTS}

This section presents the simulation results for the proposed localization algorithm and compares them to those of the algorithms reported in [18]-[20]. The Monte Carlo simulation is configured to perform 500 runs for each simulation point, where a PSO with 12 particles and 10 generations is applied to find the global maximum of the log-likelihood function. The RFID tags with known positions are uniformly distributed over a grid with an area of $(200 \mathrm{~m} \times 200 \mathrm{~m})$. The obtained results are generated for various operating scenarios such as the reader location, SNR and number of tags. The system and channel parameters for the downlink (reader $\rightarrow$ tag) are: $\mathcal{E}\left\{\rho_{k}[l]\right\}=1 \forall k, N_{0}=10^{-10} \mathrm{~W} / \mathrm{Hz}, P_{R}=0 \mathrm{dBW}$, $P_{G}=-10 \mathrm{dBW}, \sigma_{h}^{2}=1, \lambda=0.3 \mathrm{~m}$, and $d_{0}=30 \mathrm{~m}$. The RMSE is defined as $\sqrt{\mathcal{E}\left[\left(x_{R}-\hat{x}_{R}\right)^{2}+\left(y_{R}-\hat{y}_{R}\right)^{2}\right]}$.

Fig. 3 depicts the log-likelihood function in three dimensional representation, where 256 tags are distributed over the RoI to localize an RFID reader located at $\left(x_{R}=0, y_{R}=0\right)$ position. The $\mathrm{SNR} \triangleq P_{G} / \sigma_{\varphi}^{2}$ is set to $50 \mathrm{~dB}$ and the number of RISs $L=50$. As can be noted from the figure, the loglikelihood function has a global maximum that corresponds to the Cartesian coordinates of the reader estimated location.

Fig. 4 shows the RMSE of the proposed estimator for different values of $K$ using $\mathrm{SNR}=8 \mathrm{~dB},\left(x_{R}=5, y_{R}=22\right)$ and $L=10$. The figure also presents the RMSE of the LVA [19], SDE [18], HDE [20], KNN [21], and the CRLB. The results in the figure show that the proposed LEC outperforms all the other considered estimators for the entire range of $K$. However, the improvement depends on the values of $K$. The average relative improvement over the considered range of $K$ with respect to the LVA, SDE, HDE and KNN is about $26.5 \%$, $46.1 \%, 74.3 \%$, and $26.9 \%$, respectively. ${ }^{\left[R_{2,2}\right]}$ Moreover, it can be noted that the LVA performs poorly at low values of $K$ since the correction window may include readings from 


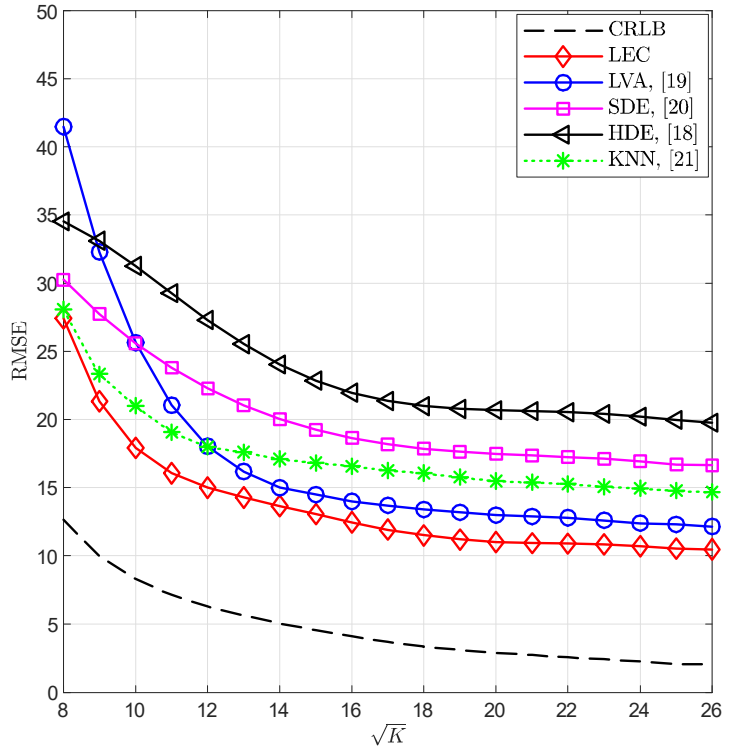

Fig. 4. RMSE of the estimated position using the proposed LEC, LVA [19], $\mathrm{SDE}[20] \mathrm{HDE}[18]$ and ${ }^{\left[R_{2,2}\right]} \mathrm{KNN}[21]$ using SNR $=8 \mathrm{~dB}$.

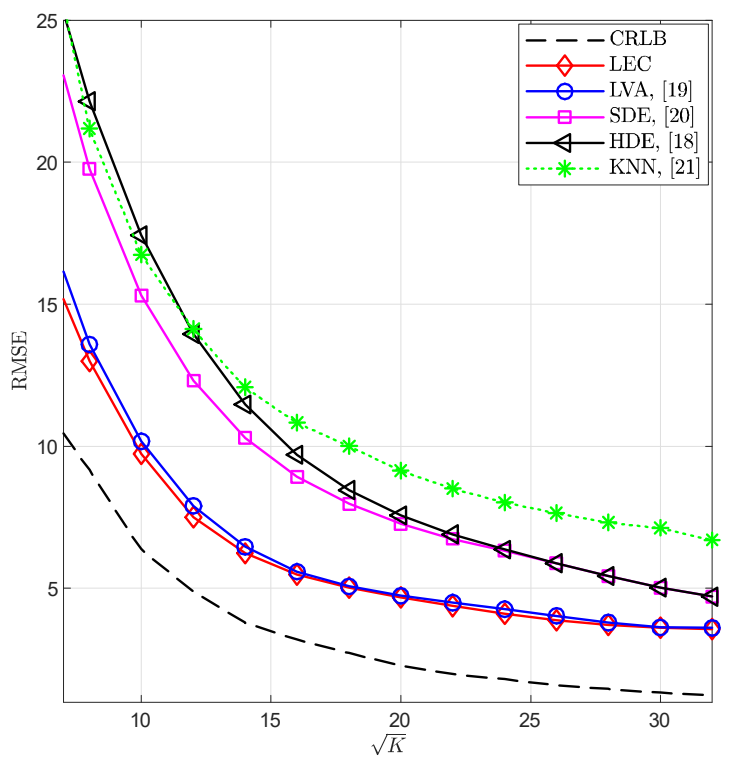

Fig. 5. RMSE of the estimated position using the proposed LEC, LVA [19], $\mathrm{SDE}[20] \mathrm{HDE}[18]^{\left[R_{2,2}\right]}$ and KNN [21] using SNR $=15 \mathrm{~dB}$.

distant tags. The KNN algorithm outperforms the HDE and SDE for the entire range of $K$, and the LVA for $K<144$. The KNN and LEC demonstrate equivalent RMSE at $K=64$.

Fig. 5 is generated using the same parameters used for Fig. 4 except that $\mathrm{SNR}=15 \mathrm{~dB}$. As can be seen from the figure, all the considered algorithms exhibit significant RMSE

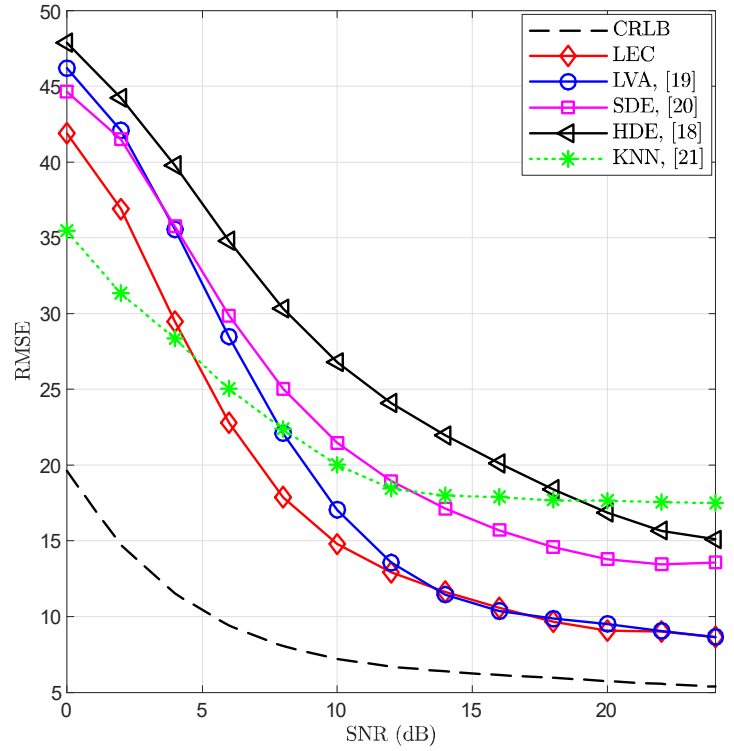

Fig. 6. The RMSE of the estimated position using the proposed LEC, LVA [19], SDE [20] HDE [18] ${ }^{\left[R_{2,2}\right]}$ and KNN [21] versus SNR, $K=100$.

reduction, particularly the LVA one since the assumption that the tag $\rightarrow$ reader channel is error free becomes plausible at such high SNRs. The average relative improvement of the proposed LEC algorithm over the LVA, SDE, HDE, and KNN algorithms is about $4.5 \%, 55.1 \%, 67.5 \%$, and $84 \%$, respectively. ${ }^{\left[R_{2,2}\right]}$ The figure also shows that the KNN RMSE improves by increasing the SNR, though at a slower rate in comparison to the other considered techniques. Moreover, the results in Figs. 4 and 5 show that the RMSE of the proposed estimator approaches the CRLB when there is a large number of tags and high SNRs.

Fig. 6 shows the RMSE of the considered estimators versus SNR using $L=10, K=100$, and the reader is located at $\left(x_{R}=10, y_{R}=27\right)$. The results in the figure show that the proposed LEC noticeably outperforms the SDE and HDE for the considered range of SNRs, and the LVA at low SNRs. For SNR $\gtrsim 12 \mathrm{~dB}$, the RMSE of the LEC and LVA converge since the tag $\rightarrow$ reader link becomes nearly error free. ${ }^{\left[R_{2,2}\right]}$ The KNN outperforms the proposed LEC at SNRs $\lesssim 4.7 \mathrm{~dB}$, which is due to the fact the error concealment process fails to improve the performance at very low SNRs. The average improvement with respect to the LVA, SDE, HDE and KNN is about $12.2 \%$, $31.5 \%, 53.5 \%$, and $24.5 \%$, respectively.

\section{VI. ${ }^{\left[R_{1,3}\right]}$ CONCLUSION AND FUTURE WORK}

In this paper, the problem of RFID reader localization using distributed tags was considered. To mitigate the impact of channel impairments, a novel maximum likelihood estimator was proposed based on the signals received from the tags. By considering that the reader performs hard decisions on the 
signals received from the tags, a simple error concealment process is applied to improve the estimator's accuracy. The system was simulated for different cases of interest and the RMSE results were compared to four benchmark estimators. The obtained results showed that the proposed estimator considerably outperforms the other considered localization techniques. Moreover, the CRLB for the RMSE of the proposed algorithm was derived to asses its efficiency.

$\left[R_{1,3}\right]$ To capture the impact of various practical imperfections, evaluating the performance of the proposed algorithm experimentally is indispensable. Therefore, our future work includes developing a testbed to collect a large set of results in different channel conditions and compare the experimental results with the simulation and analytical results obtained in this work.

\section{REFERENCES}

[1] A. Athalye, V. Savic, M. Bolic, and P. Djuric, "Novel semi-passive RFID system for indoor localization,” IEEE Sensors J., vol. 13, no. 2, pp. 528537, Feb. 2013.

[2] ${ }^{\left[R_{2}, 4\right]}$ W. Zhu, J. Cao, Y. Xu, L. Yang and J. Kong, "Fault-tolerant RFID reader localization based on passive RFID tags," IEEE Trans. Parallel Distrib. Syst., vol. 25, no. 8, pp. 2065-2076, Aug. 2014.

[3] H. Yu, J. Chen, and T. Hsiang, "Design and implementation of a realtime object location system based on passive RFID tags," IEEE Sensors $J$. , vol. 15, no. 9, pp. 5015-5023, Sep. 2015.

[4] L. Xie, Y. Yin, A. Vasilakos, and S. Lu, "Managing RFID data: challenges, opportunities and solutions," IEEE Commun. Surveys Tuts., vol. 16, no. 3, pp. 1294-1311, 2016.

[5] E. DiGiampaolo and F. Martinelli, "Mobile robot localization using the phase of passive UHF RFID signals," IEEE Trans. Ind. Electron., vol. 61, no. 1, pp. 365-376, Jan. 2014.

[6] Z. Zhang, et al., "Item-level indoor localization with passive UHF RFID based on tag interaction analysis," IEEE Trans. Ind. Electron., vol. 61, no. 4, pp. 2122-2135, Apr. 2014

[7] ${ }^{\left[R_{2,4}\right]}$ M. Scherhäufl, M. Pichler, and A. Stelzer, "UHF RFID localization based on evaluation of backscattered tag signals," IEEE Trans. Instrum. Meas., vol. 64, no. 11, pp. 2889-2899, Nov. 2015.

[8] Y. Zhao, et al., "Similarity analysis-based indoor localization algorithm with backscatter information of passive UHF RFID tags," IEEE Sensors J., vol. 17, no. 1, pp. 185-193, Jan. 2017.

[9] S. Subedi, E. Pauls, and Y. Zhang, "Accurate localization and tracking of a passive RFID reader based on RSSI measurements," IEEE Trans. Radio Freq. Identification, vol. 1, no. 2, pp. 144-154, Jun. 2017.

[10] ${ }^{\left[R_{2,4}\right]}$ H. Xiong, J. Tang, H. Xu, W. Zhang, and Z. Du, "A robust single GPS navigation and positioning algorithm based on strong tracking filtering," IEEE Sensors J., vol. 18, no. 1, pp. 290-298, Jan. 2018.

[11] C. Yao and W. Hsia, "An indoor positioning system based on the dualchannel passive RFID technology," IEEE Sensors J., vol. 18, no. 11, pp. 4654-4663, 1 Jun. 2018.

[12] W. Shi, et al., "Optimizing directional reader antennas deployment in UHF RFID localization system by using a MPCSO algorithm," IEEE Sensors J., vol. 18, no. 12, pp. 5035-5048, Jun. 2018.

[13] ${ }^{\left[R_{2}, 4\right]}$ M. El-Absi, A. Abbas, A. Abuelhaija, F. Zheng, K. Solbach, and T. Kaiser, "High-accuracy indoor localization based on chipless RFID systems at THz band," IEEE Access, vol. 6, pp. 54355-54368, Sep. 2018.

[14] $\left.{ }^{\left[R_{2}, 4\right.}\right]$ J. Zhang, Y. Lyu, J. Patton, S. Periaswamy, and T. Roppel, "BFVP A probabilistic UHF RFID tag localization algorithm using Bayesian filter and a variable power RFID model," IEEE Trans. Ind. Electron., vol. 65 , no. 10 , pp. 8250-8259, Oct. 2018.

[15] $\left.{ }^{\left[R_{2}, 4\right.}\right]$ H. Xiong, et al., "Efficient bias reduction approach of time-offlight-based wireless localization networks in NLOS states," IET Radar Sonar Nav., vol. 12, no. 11, pp. 1353-1360, Nov. 2018.

[16] ${ }^{\left[R_{2,4}\right]}$ H. Xiong, M. Peng, S. Gong, and Z. Du, "A novel hybrid RSS and TOA positioning algorithm for multi-objective cooperative wireless sensor networks," IEEE Sensors J., vol. 18, no. 22, pp. 9343-9351, Nov. 2018

[17] ${ }^{\left[R_{2,4}\right]}$ M. Scherhäufl, B. Rudić, A. Stelzer, and M. Pichler-Scheder, "A blind calibration method for phase-of-arrival-based localization of passive UHF RFID transponders," IEEE Trans. Instrum. Meas., vol. 68, no. 1, pp. 261-268, Jan. 2019.

[18] O. Ozdemir, R. Niu, and P. Varshney, "Channel aware target localization with quantized data in wireless sensor networks," IEEE Trans. Signal Process., vol. 57, no. 3, pp. 1190-1202, Mar. 2009.

[19] N. Katenka, E. Levina, and G. Michailidis, "Tracking multiple targets using binary decisions from wireless sensor networks," J. Am. Stat. Assoc., vol. 108, no. 502, pp. 398-410, Feb. 2013.

[20] R. Niu, A. Vempaty, and P. Varshney, "Received-signal-strength-based localization in wireless sensor network," Proc. IEEE, vol. 106, no. 7, pp. 1166-1182, Jul. 2018.

[21] M. Hoang, et al., "A soft range limited K-nearest neighbors algorithm for indoor localization enhancement," IEEE Sensors J., vol. 18, no. 24, pp. 10208-10216, Dec. 2018.

[22] M. Anisetti, C. Ardagna, V. Bellandi, E. Damiani, and S. Reale, "Mapbased location and tracking in multipath outdoor mobile networks," IEEE Trans. Wireless Commun., vol. 10, no. 3, pp. 814-824, Mar. 2011.

[23] M. Fernandez and S. Williams, "Closed-form expression for the Poisson-binomial probability density function," IEEE Trans. Aerosp. Electron. Syst., vol. 46, no. 2, pp. 803-817, Apr. 2010.

[24] H. Poor. An introduction to signal detection and estimation, 2nd ed. New York: Springer-Verlag, 1994.

[25] $\left[R_{1,1 a}\right]$ K. Wei, Z. Zhang, Z. Feng, and M. Iskander, "A MNG-TL loop antenna array with horizontally polarized omnidirectional patterns," IEEE Trans. Antennas Propag., vol. 60, no. 6, pp. 2702-2710, Jun. 2012.

[26] $\left[R_{1,1 a}\right]$ J. Shi, X. Wu, X. Qing, and Z. Chen, "An omnidirectional circularly polarized antenna array," IEEE Trans. Antennas Propag., vol. 64, no. 2, pp. 574-581, Feb. 2016.

[27] $\left[R_{1,1 a}\right]_{\mathrm{K}}$. Wei, Z. Zhang, and Z. Feng, "Design of a wideband horizontally polarized omnidirectional printed loop antenna," IEEE Antennas Wireless Propag. Lett., vol. 11, pp. 49-52, 2012

[28] ${ }^{\left[R_{1,2}\right]}$ S. Chen, K. Lin, and R. Mittra, "Miniature and near-3D omnidirectional radiation pattern RFID tag antenna design," Electron. Lett., vol. 45, no. 18, pp. 923-924, 27 August 2009.

\begin{tabular}{|c|} 
\\
\\
PLACE \\
PHOTO \\
HERE \\
\end{tabular}

M ohammad A. Al-Jarrah, received his MSc. degree in Electrical Engineering/Wireless Communications from Jordan University of Science and Technology (JUST) in 2011. From Sep. 2011 to Mar. 2017, he had been working as a lecturer at Prince Sattam bin Abdulaziz University (PSAU), Saudi Arabia. Currently, he is working as a Lab instructor at Khalifa University (KU), United Arab Emirates. His current research area includes: Distributed Decision Fusion Systems, Statistical Signal Processing, Target Tracking in Wireless Sensor Networks, RFID Communications, Cognitive Radio Networks, Visible Light Communication (VLC), Cooperative Communications, and Orthogonal Frequency Division Multiplexing (OFDM). 


\section{Page 9 of 14}

A rafat Al-Dweik (S'97-M'01-SM'04) received the B.Sc. degree in Telecommunication Engineering from Yarmouk University, Jordan, in 1994 and the M.S. (Summa Cum Laude) and Ph.D. (Magna Cum Laude) degrees in electrical engineering from Cleveland State University, Cleveland, OH, USA in 1998 and 2001, respectively. He was with Efficient Channel Coding, Inc., Cleveland-Ohio, from 1999 to 2001, where he was a Research and Development Engineer working on advanced modulation, coding, and synchronization techniques. From 2001 to 2003, he was the Head of Department of Information Technology at the Arab American University in Palestine. From 2003 to 2012, he was with the Communications Engineering Department, Khalifa University (KU), United Arab Emirates. He joined University of Guelph, ON, Canada, as an Associate Professor from 2013-2014. Dr. Al-Dweik is a Visiting Research Fellow at the School of Electrical, Electronic and Computer Engineering, Newcastle University, Newcastle upon Tyne, UK since 2006 till present. He is also a Research Professor and member of the School of Graduate Studies at Western University, London, ON, Canada. Dr Al-Dweik has extensive editorial experience where he serves as an Associate Editor at the IEEE Transactions on Vehicular Technology and IET Communications. Moreover, he was a TPC member in several major conferences such as the IEEE GLOBECOM, ICC, PIMRC and WCNC. Dr Al-Dweik has received several research awards and he was a recipient of the Fulbright Scholarship.

\begin{tabular}{|c|}
\hline \\
PLACE \\
PHOTO \\
HERE \\
\end{tabular}

E mad Alsusa (M'06-SM'07) received the Ph.D. degree in electrical and electronic engineering from the University of Bath, Bath, U.K., in 2000. He then joined the University of Edinburgh, Edinburgh, U.K., as a Mobile VCE Postdoctoral Research Fellow, working on link enhancement techniques for future high-data-rate wireless communication systems. In 2003, he joined The University of Manchester, Manchester, U.K., as an academic member of the School of Electrical and Electronic Engineering, where he lectures on communication engineering. His research interests include signal processing techniques and the analysis of wireless communication networks, with particular focus on cognitive radio, interference mitigation, multiuser multiple-input multipleoutput, green communications, and energy and spectrum optimization techniques. Dr. Alsusa has served as a Technical Program Committee Member for numerous IEEE flagship conferences and chaired the Manchester EEE postgraduate conference in 2010

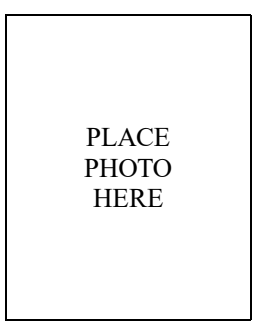

E rnesto Damiani holds a $\mathrm{PhD}$ from Universita' degli Studi di Milano, where he leads the SEcure Service-oriented Architectures Research (SESAR) Lab. Ernesto is the Founding Director of the Center for Cyber-Physical Systems at Khalifa University, in the UAE. He received a honorary doctorate from Institut National des Sciences Appliquées de Lyon, France (2017) for his contributions to research and teaching on Big Data analytics. Ernesto's research spans Cyber-security, Big Data and cloud/edge processing, where he has published over 600 peerreviewed articles and books. He is Distinguished Scientist of ACM and a recipient of the 2017 Stephen Yau Award. 